\title{
Looking for variable stars in galactic open clusters
}

\author{
Claudia Greco ${ }^{1}$, Nami Mowlavi ${ }^{1}$, Laurent Eyer ${ }^{1}$, Maxime Spano $^{1}$, \\ Mihaly Varadi ${ }^{1}$ and Gilbert Burki ${ }^{1}$ \\ ${ }^{1}$ Geneva Observatory, University of Geneva, ch. des Maillettes 51, CH-1290, Sauverny, \\ Switzerland \\ email: claudia.greco@unige.ch
}

\begin{abstract}
A long-term project, aiming at systematic search for variable stars in Galactic Open Clusters (OCs), was started at the Geneva Observatory in 2002. We have been observing regularly a sample of twenty-seven Galactic Open Clusters in the $U, B, V$ Geneva filters (hereafter $U, B, V)$. The goal is to identify and to study their variable stars, as well as the connection between the variable stars in a cluster and the cluster properties. We present the status of this work in progress, and show preliminary results for one of these clusters, IC 4651.
\end{abstract}

Keywords. open clusters and associations: general, individual (IC 4651); stars: variable

\section{Introduction}

Long-term observations of a sample of twenty-seven Galactic OCs have been carried out in $U, B$ and $V$ (see Cherix et al., 2006). Twelve OCs in the Southern Hemisphere have been observed with Euler-Cam, mounted on the $1.2 \mathrm{~m}$ Euler Swiss Telescope at La Silla, Chile and fifteen OCs in the Northen Hemisphere OCs with Merope-Cam installed on the $1.2 \mathrm{~m}$ Mercator Belgian telescope, in La Palma, Canary Islands, Spain. The field of view of the two cameras (Euler: 11.5' $\times 11.5^{\prime}$; Mercator: $6.5^{\prime} \times 6.5^{\prime}$ ) is centered on the cluster and contains cluster stars as well as Milky Way field stars.Data acquisition and data reduction are still in progress. For the Southern clusters we already collected more than $200 \mathrm{U}, 200 \mathrm{~B}$ and $2000 \mathrm{~V}$ images on a time baseline of 7 years. In Table 1 , we summarize some properties for the OCs included in this project, includeing coordinates, estimated age and metallicities. Times series for Northern OCs include so far almost 1600 $\mathrm{V}$ image over a 5 year baseline.

The goal of the project is to map the whole variable star population in each cluster in our database. Stellar variability provides independent measurements for the physical parameters of OCs.

\section{Data Reduction}

We developed a semi-automatic pipeline to prereduce and reduce all the images. We perform PSF photometry with the DaophotII/Allstar (Stetson 1998, 1999) packages. For each image, we build a proper PSF, using at least one hundred selected stars chosen among the brightest and most isolated ones. All the reduced images are then aligned to a common reference list of stars in order to build the Color Magnitude Diagram and the time series for each star. Time series are then searched for objects showing variability. The time series of the candidate variables have been studied using two different codes. We used GrATis (Graphical Analyzer of Time Series; Di Fabrizio 1999; Clementini 2000) and Period04 (Lenz \& Breger 2005). 

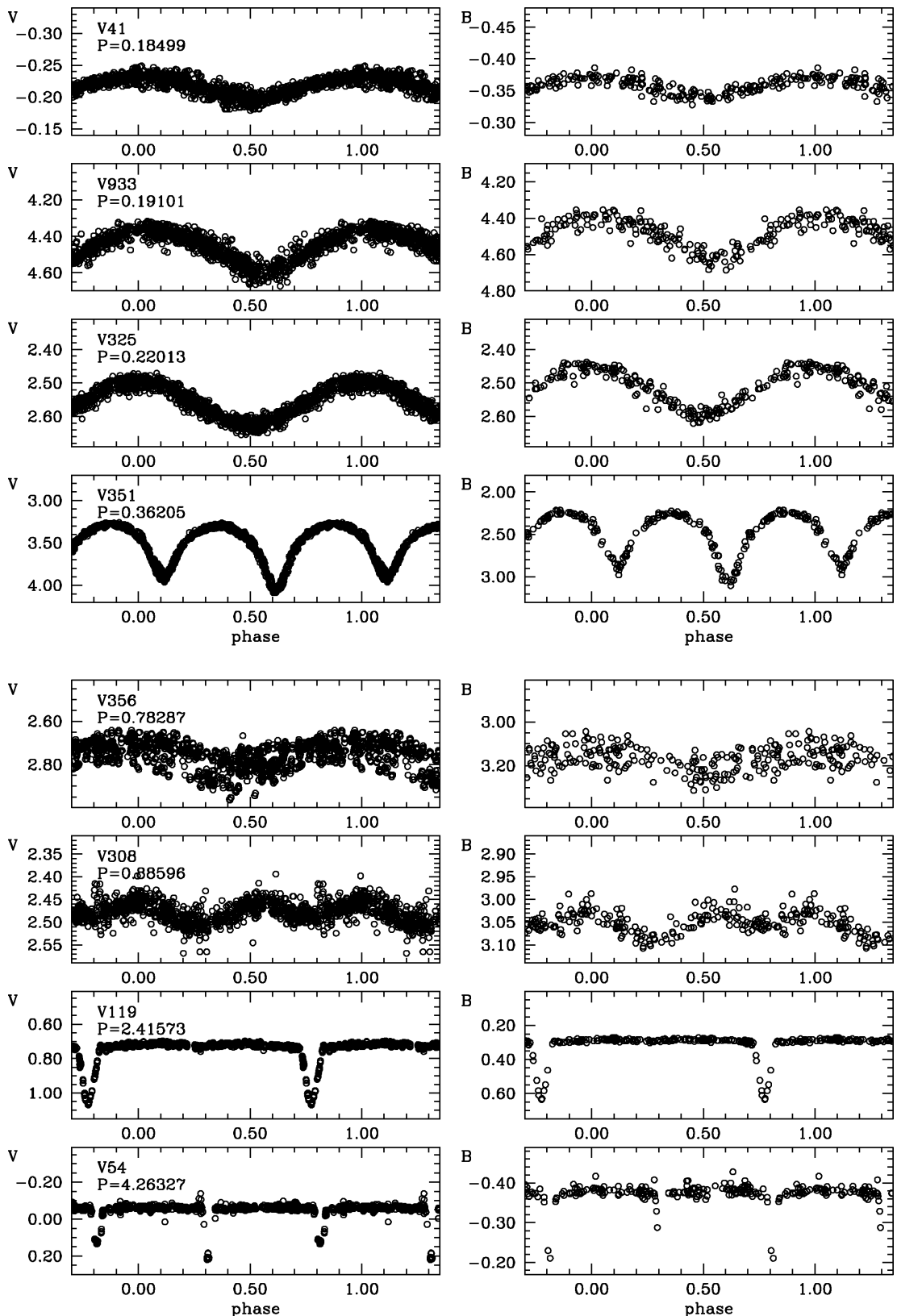

Figure 1. Differential magnitude folded light curves for the monoperiodic variable stars we identified in IC 4651. Periods are in days. Period of the variability increase from top to bottom.

The first open cluster to be analyzed has been IC 4651. IC 4651 is a quite old and metal rich open cluster. We found variable stars with periods spanning from 0.18 to more than 53 days, including bona fide $\delta$ Scuti and binary stars. Several candidates show multi-periodic variability and variation of the period along the time series. In Fig. 1 we show differential magnitude folded light curves for the monoperiodic variable stars we identified. 
Table 1. Basic information about the twenty-seven OCs in the Geneva database. In bold, the eight clusters we have already reduced. References to previous works from the same database are in footnotes. Literature data are taken from WEBDA.

\begin{tabular}{|c|c|c|c|c|c|}
\hline Open Cluster & RA & DEC & $\log ($ age $)$ & $\langle[\mathrm{Fe} / \mathrm{H}]\rangle$ & Telescope \\
\hline & $(\mathrm{J} 2000)$ & $(\mathrm{J} 2000)$ & (years) & $(\mathrm{dex})$ & \\
\hline NGC 3766 & $11: 36: 14$ & $-61: 36: 30$ & 7.160 & - & Euler \\
\hline NGC 4103 & $12: 06: 40$ & $-61: 15: 00$ & 7.393 & - & Euler \\
\hline NGC 6755 & $19: 07: 49$ & $+04: 16: 00$ & 7.719 & - & Mercator \\
\hline NGC 7654 & $23: 24: 48$ & $+61: 35: 36$ & 7.764 & - & Mercator \\
\hline NGC 7039 & $21: 10: 48$ & $+45: 37: 00$ & 7.280 & - & Mercator \\
\hline $\mathrm{NCG} 5617^{1}$ & $14: 29: 44$ & $-60: 42: 42$ & 7.915 & - & Euler \\
\hline NGC 6996 & $20: 56: 30$ & $-54: 13: 06$ & 8.000 & - & Euler \\
\hline NGC6067 & $16: 13: 11$ & $-54.13: 06$ & 8.076 & 0.13 & Euler \\
\hline NGC3247 & $10: 25: 51$ & $-57: 55: 24$ & 8.083 & - & Euler \\
\hline NGC 2323 & $07: 02: 42$ & $-08: 23: 00$ & 8.096 & 0.02 & Euler \\
\hline NGC 1513 & $04: 09: 57$ & $+49: 30: 54$ & 8.110 & - & Mercator \\
\hline NGC 6705 & $18: 51: 05$ & $-06: 16: 12$ & 8.302 & 0.13 & Mercator \\
\hline NGC2437 & $07: 41: 46$ & $-14: 48: 36$ & 8.390 & 0.05 & Euler \\
\hline NGC3532 & $11: 05: 39$ & $-58: 45: 12$ & 8.492 & -0.02 & Euler \\
\hline NGC 2194 & $06: 13: 45$ & $+12: 48: 24$ & 8.515 & - & Mercator \\
\hline NGC 1907 & $05: 28: 05$ & $+35: 19: 30$ & 8.567 & - & Mercator \\
\hline NGC2447 & $07: 44: 30$ & $-23: 51: 24$ & 8.588 & 0.03 & Euler \\
\hline NGC 2324 & $07: 04: 07$ & $+01: 02: 42$ & 8.630 & -0.52 & Mercator \\
\hline NGC 1245 & $03: 14: 41$ & $+47: 14: 12$ & 8.704 & 0.10 & Mercator \\
\hline NGC 6811 & $19: 37: 17$ & $+46: 23: 18$ & 8.799 & - & Mercator \\
\hline NGC $1901^{2}$ & $05: 18: 15$ & $-68: 26: 12$ & 8.920 & - & Euler \\
\hline NGC 6134 & $16: 27: 46$ & $-49: 09: 06$ & 8.968 & 0.18 & Euler \\
\hline NGC 2420 & $07: 38: 23$ & $+21: 34: 24$ & 9.048 & -0.26 & Mercator \\
\hline IC 4651 & $17: 24: 49$ & $-49: 56: 00$ & 9.057 & 0.10 & Euler \\
\hline NGC 7789 & $23: 57: 24$ & $+56: 42: 30$ & 9.235 & -0.08 & Mercator \\
\hline NGC 6939 & $20: 31: 30$ & $+60: 39: 42$ & 9.346 & 0.02 & Mercator \\
\hline NGC 188 & $00: 47: 28$ & $+85: 15: 18$ & 9.632 & -0.02 & Mercator \\
\hline
\end{tabular}

Notes:

1 see Carrier 2009

2 see Cherix 2006

\section{Conclusions}

An overall accuracy of a few mmag, plus the long time baseline, allows the identification of various kinds of variable stars, including small period and small amplitude ones. The analysis of the variable stars found in IC 4651 indeed confirms the potentialities of this project to identify variable stars and to use them to characterize the properties of OCs.

\section{References}

Carrier, F. et al. 2009, ArXiv e-prints, 0901.0627.

Cherix, M., Carrier, F., Burki, G., \& Blecha, A. 2006, Memorie della Societa Astronomica Italiana, 77, 328-+

Clementini, G., et al. 2000, AJ, 120, 2054-2064

Dias, W. S., Alessi, B. S., Moitinho, A., \& Lépine, J. R. D. 2002, A\& A, 389, 871-873

Di Fabrizio, L. 1999, Master Thesis, Universitá di Bologna, Dipartimento di Astronomia, via Ranzani 1

Lenz, P. \& Breger, M. 2005, Communications in Asteroseismology 146, 53-136

Lynga, G. 1987, Catalogue of Open Cluster Data (5th Ed.), Lund Observ., Solvegatan 27, Lund Stetson, P. B. 1996, PASP, 108, 851

Stetson, P. B., Hesser, J. E., \& Smecker-Hane, T. A. 1998, PASP, 110, 533-552 